Supporting Information

\title{
An Optical Microfiber Biosensor for CEACAM5 \\ Detection in Serum: Sensitization by a Nanosphere Interface
}

Aoxiang Xiao, Yunyun Huang*, Jiaying Zheng, Pengwei Chen, Bai-Ou Guan

Guangdong Provincial Key Laboratory of Optical Fiber Sensing and Communications, Institute of Photonics Technology, Jinan University, Guangzhou 511486, China

AUTHOR INFORMATION

Corresponding Author

* Address correspondence to: Yunyun Huang, email: thuangyy6@jnu.edu.cn

KEYWORDS. Early detection, optical microfiber, biosensor, interfacial sensitization, in serum. 


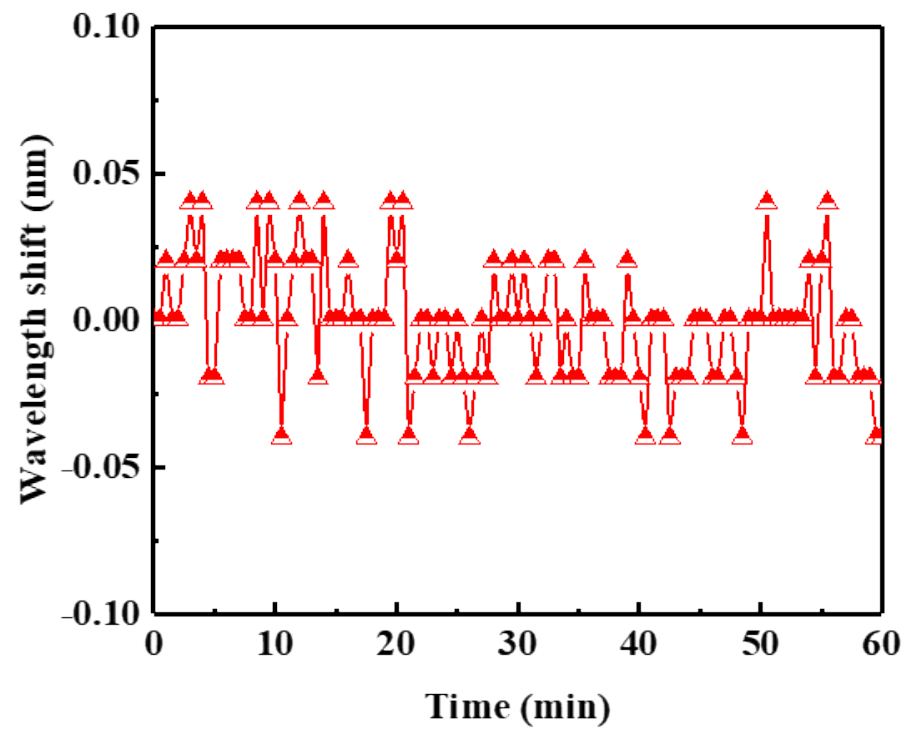

Figure S1. The wavelength stability of the as-prepared sensor in PBS solution. 

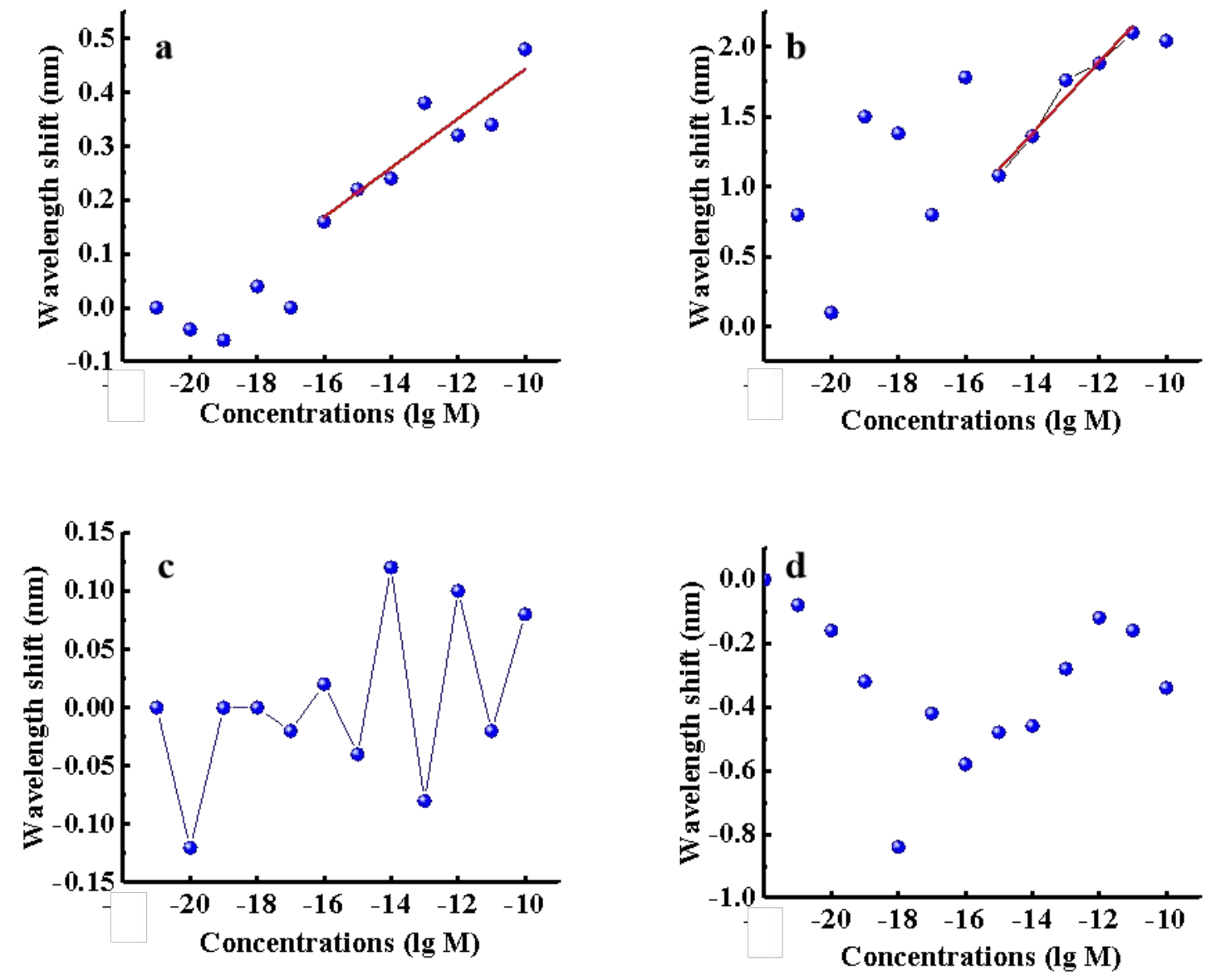

Figure S2. Wavelength shifts of control microfiber sensor with different nanointerfaces (a. The PS@Au interface without antibodies, sensitivity: $0.046 \mathrm{~nm} / \mathrm{lg} \mathrm{M}$; b. The PS interface with antibodies but without $\mathrm{Au}$ NPs, sensitivity: $0.256 \mathrm{~nm} / \mathrm{lg} \mathrm{M}$; c. The Au NPs interface directly functionalized on the microfiber without antibodies or PS nanospheres; d. The mixture interface without good PS/Au contact (the PS nanospheres with antibodies were functionalized on microfiber first, the Au NPs added to the microfiber surface following)). 


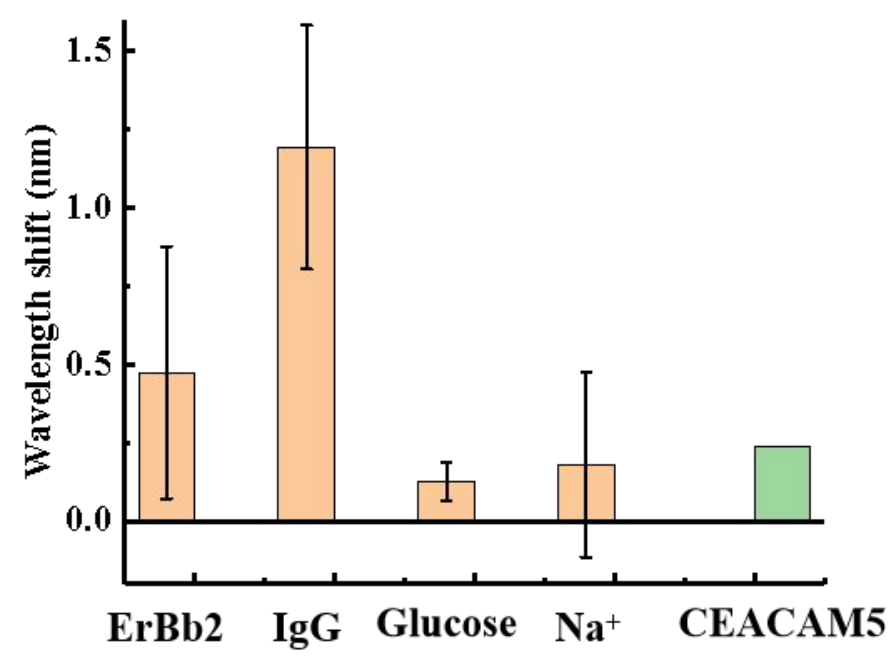

Figure S3. Optical responses of the one of the control sensors without antibodies (the sensor with PS@Au interface without antibodies) versus CEACAM5 at a concentration of $10^{-14} \mathrm{M}$ in PBS solution, and other interfering molecules at high concentrations in PBS solution (glucose and $\mathrm{NaCl}$ : $10^{-6} \mathrm{M}$; ErbB2: 10-9 M; IgG: $\left.10^{-9} \mathrm{M}\right)$

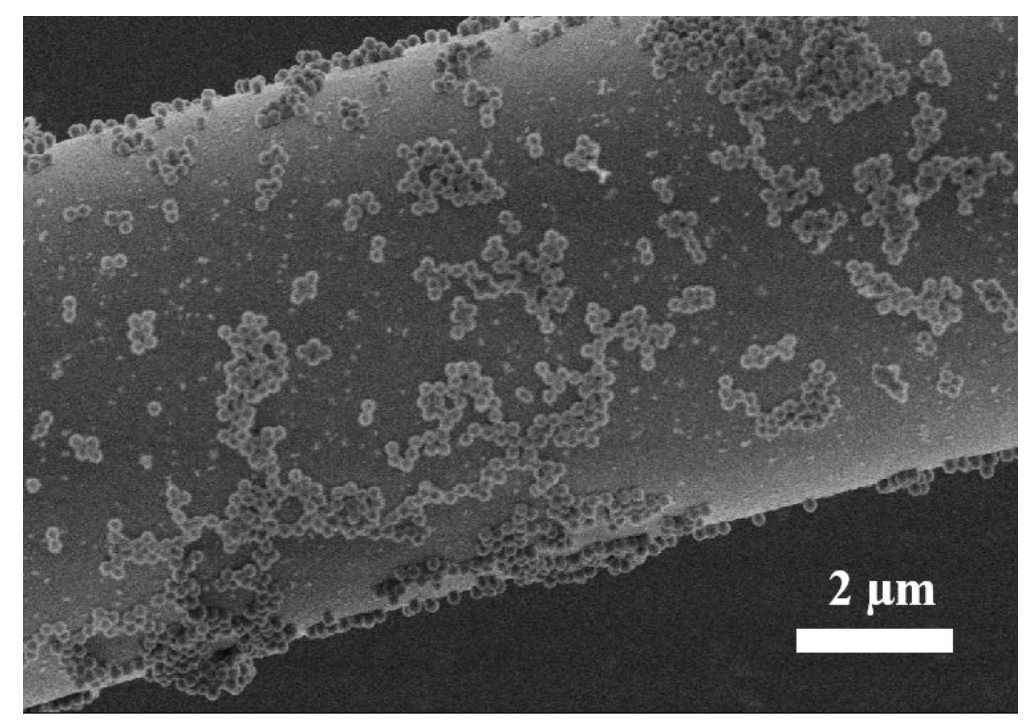

Figure S4. The SEM images of microfiber surface after detection. 


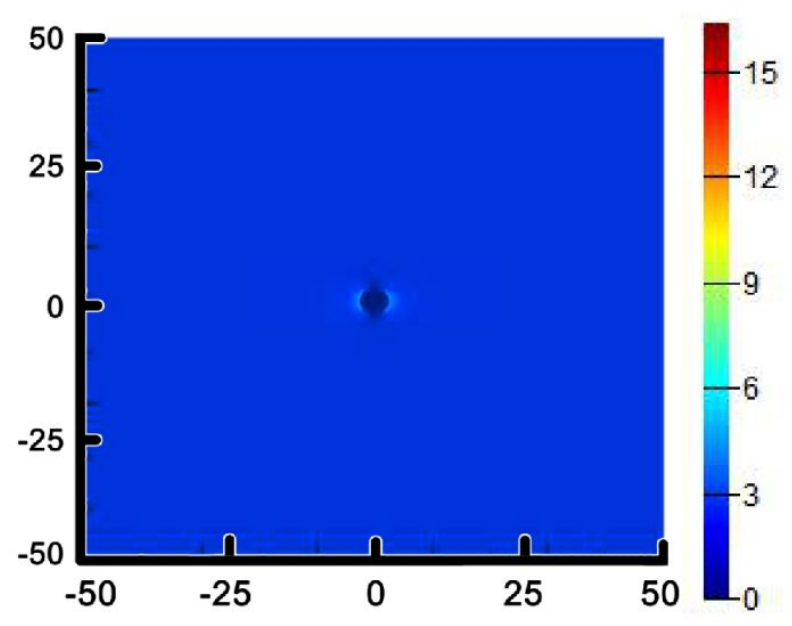

Figure S5. Near-field intensity mapping of the microfiber surface functionalized by individual gold nanoparticle.

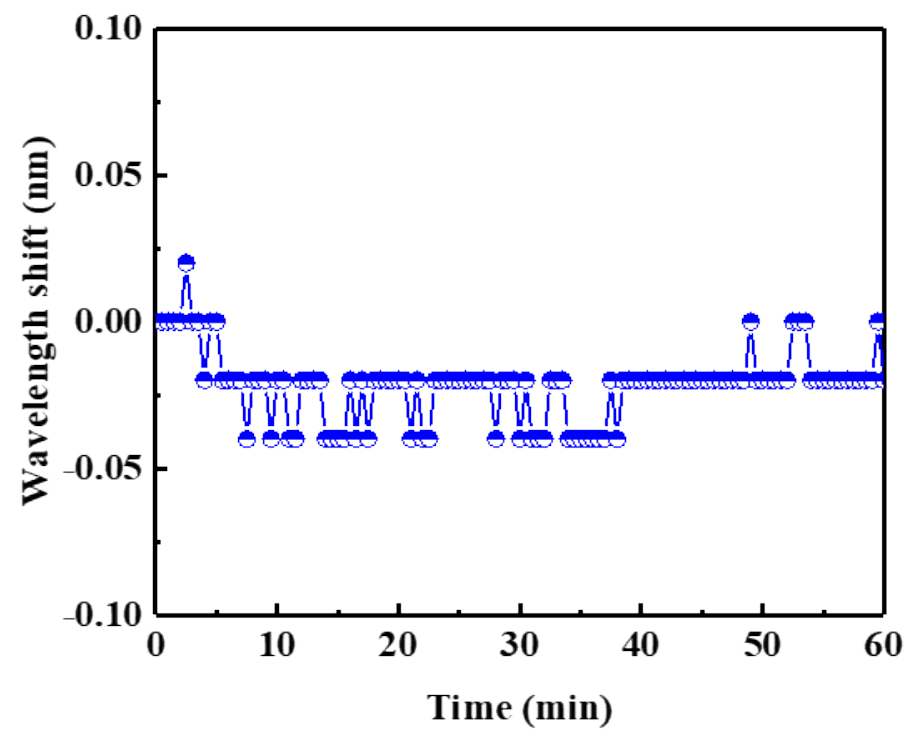

Figure S6. The wavelength stability of the as-prepared sensor in 10\% serum solution. 